\title{
Estudo da Relação entre a Variabilidade dos Índices de Vegetação e Temperatura da Região Nordeste do Brasil
}

\author{
Aldenia Ribeiro dos Santos Gomes ${ }^{1}$ (D), José Maria Brabo Alves ${ }^{2}$, Emerson Mariano da Silva ${ }^{2}$, \\ Micaelle Ribeiro dos Santos Gomes ${ }^{3}$, Camila Ribeiro dos Santos Gomes ${ }^{4}$ \\ ${ }^{1}$ Curso de Mestrado Profissional em Climatologia e Aplicações em Países da CPLP e África, \\ Universidade Estadual do Ceará, Fortaleza, CE, Brasil. \\ ${ }^{2}$ Departamento de pós-graduação em Climatologia e Aplicações em Países da CPLP e África, \\ Universidade Estadual do Ceará, Fortaleza, CE, Brasil. \\ ${ }^{3}$ Curso de Graduação em Química, Universidade Estadual do Ceará, Fortaleza, CE, Brasil. \\ ${ }^{4}$ Curso de Graduação em Biologia, Universidade Estadual do Ceará, Fortaleza, CE, Brasil.
}

Recebido em: 28 de Abril de 2019 - Aceito em: 24 de Maio de 2019

\begin{abstract}
Resumo
Esse estudo teve por objetivo investigar a inter-relação entre a variabilidade de índices de vegetação (NDVI - Índice de Vegetação de Diferença Normalizada, VCI - índice de condição da vegetação) e temperatura (TCI - Índice de Condição de Temperatura) sobre o Nordeste do Brasil, utilizando dados de sensoriamento remoto, banco de dados NOAA-NESDIS, obtidos na série histórica de 1982 a 2011. Foram feitas algumas inferências acerca da variação desses índices de vegetação e temperatura, associada as mudanças climáticas, em períodos sazonais e anos de contrastes climáticos sob a influência de El Niños, La Niñas e condições Normais do Pacífico. Os dados de saída das compilações das rotinas Fortran foram disponibilizados em gráficos e gerados pelo software GrADS (Grid Analysis and Display System). Os resultados mostraram forte correlação positiva entre os indices de NDVI associado ao VCI e TCI na região semiárida do NEB. Esse resultado foi devido aos percentuais de precipitação baixos que esta região possui, tornando mais evidente a forte relação destes índices de vegetação e temperatura no semiárido do NEB. Já para outras áreas da região do NEB que possuem maiores índice de precipitação, a relação entre o NDVI e a precipitação não foi tão evidente neste estudo.
\end{abstract}

Palavras-chave: estresse vegetativo, variabilidade térmica, sensoriamento remoto.

\section{Study of the Relationship Between the Variability of Vegetation and Temperature Indexes of the Northeast Region of Brazil}

\begin{abstract}
The objective of this study was to investigate the interrelation between vegetation index variability (NDVI - Normalized Difference Vegetation Index, VCI - vegetation condition index) and temperature (TCI - Temperature Condition Index). on the Northeast of Brazil, using remote sensing data, NOAA-NESDIS database, obtained in the historical series from 1982 to 2011. Some inferences were made about the variation of these indices of vegetation and temperature, associated with climatic changes, in periods seasonal and in years of climatic contrasts under the influence of El Niños, La Niñas and Pacific Normal Conditions. The output data of the compilations of the Fortran routines were made available in graphs and generated by the GridS (Grid Analysis and Display System) software. The results showed a strong positive correlation between NDVI indices associated with IVC and TCI in the NEB semi-arid region. This result is due to the low percentages of precipitation that this region possesses, being thus more evident the strong relation with these indices of vegetation and temperature in the NEB semi-arid. As for other areas of the NEB region that have the highest precipitation index, the relationship between NDVI and precipitation was not as evident in this study.
\end{abstract}

Keywords: vegetative stress, thermal variability, remote sensing.

Autor de correspondência: Aldenia Ribeiro dos Santos Gomes, aldeniarsg@gmail.com. 


\section{Introdução}

Segundo Araújo (2011) a região Nordeste do Brasil possui uma área de $1.561 .177,8 \mathrm{~km}^{2}$, dos quais $841.260,9$ $\mathrm{km}^{2}$ abrangem o semiárido nordestino. Possui grande variabilidade de precipitação e altas taxas de evaporação, essas características mantém a região submetida frequentemente aos efeitos de secas e estiagens, com precipitação média anual entre $400 \mathrm{~mm}$ a $800 \mathrm{~mm}$ (milímetro). Segundo Uvo e Berndtsson (1996). Existem vários mecanismos físicos e sistemas atmosféricos que interagem e influenciam o regime de chuva no Nordeste e sua variabilidade térmica, citam-se o fenômeno El Niño-Oscilação Sul (ENOS), gradientes de anomalias de Temperatura da superfície do mar (TSM) no Atlântico Tropical, Ventos Alísios, Zona de Convergência Intertropical (ZCIT) no oceano Atlântico e Vórtices Ciclônicos de Altos Níveis (VCAN). No Nordeste do Brasil (NEB) existem diferentes regimes de chuvas. Na parte norte do NEB a estação chuvosa ocorre entre os meses de março a maio. Segundo Ferreira (1996) a presença de ZCIT provoca baixas pressões na região, favorecendo o desenvolvimento de nuvens do tipo cumulonimbus na costa. Na parte sul e sudeste do NEB a estação chuvosa ocorre durante os meses de dezembro a fevereiro, devido à influência das frentes frias vindas do sul nos meses de novembro a fevereiro. Na costa e na parte leste do NEB a estação chuvosa ocorre principalmente entre maio a julho (Rao,1993).

O ENOS está associado diretamente com a variabilidade das chuvas que ocorre nos meses de janeiro, fevereiro, abril e maio, porém o El Niño também contribui para o aumento de chuvas no sul do Brasil, parte do Sudeste e do Centro-Oeste. Já o fenômeno La Niña corresponde ao inverso, representa o esfriamento anormal das águas do oceano Pacífico devido às forças dos ventos alísios. No Brasil esse fenômeno provoca efeitos opostos ao EL Niño, com a intensificação das chuvas no Nordeste e temperaturas mais amenas na Amazônia, partes do Sudeste além de queda das temperaturas na América do Norte e parte da Europa. Nos anos que ocorrem grande intensidade do fenômeno ENOS, resultam em seca e em geral aumento de temperatura no semiárido nordestino segundo (Chiang, 2000).

O NOAA (National Oceanic and Atmospheric Administration), de origem americana tem sido de grande importância para a meteorologia. Essa agência mantém satélites que possuem um sensor remoto que corresponde a um sistema opto-eletrônico que gera imagens e informações de objetos distantes. O NOAA utiliza imagens na banda visível e infravermelha e sistema de sensores modificado com filtro e chips, sensíveis à radiação e possui órbita heliossíncrona circular de $850 \mathrm{~km}$. O AVHRR-3/ NOAA (Advanced Very High Resolution Radiometer) é um sensor a bordo, com grande resolução para aplicações em meteorologia, pois utiliza um radiômetro imageador de varredura mecânica, que pode trabalhar com seis bandas espectrais. A resolução temporal do AVHRR-3/NOAA é muito maior que outros satélites de sensoriamento remoto próprio para fins meteorológicos (INPE, 2005).

Kogan (1997) em seu trabalho Global Drought Watch from Space utilizou um aplicativo global de monitoramento chamado VCI (índice de condição da vegetação) e TCI (Índice de Condição de Temperatura) na detecção de seca e avaliação de sua duração. Sua pesquisa analisou a intensidade e a cobertura de área da vegetação impactada pela seca, utilizando à coleta e análise do solo em diferentes ambientes ecológicos para calcular o estresse vegetativo causado pelos diversos tipos de seca. Os valores médios de VCI e TCI utilizados foram validados através da comparação com os dados de medições in situ em seu trabalho. Foram utilizados Algoritmos para reduzir o ruído e ajustar os dados de NDVI e BT (Temperatura de brilho). Os resultados obtidos com essa metodologia foram utilizados para quantificar o impacto da seca na produção agrícola na América do sul, na África, na Ásia, na América do Norte e Europa.

Este estudo buscou analisar a variabilidade de índices de vegetação e temperatura no NEB, avaliar as relações dos índices de vegetação e variações de temperatura e de estresse de umidade em áreas específicas no NEB; Analisar as características das variações dos índices de vegetação e variações de temperatura e estresse de umidade em áreas específicas no NEB e foram feitas também em anos de contrastes climáticos para os períodos de El Niño, La Niña e anos Normais.

\section{Materiais e Métodos}

As bases de dados de índices de vegetação e de temperatura são provenientes de satélites (dados mensais) do banco de dados da NOAA-NESDIS período de 1982-2011 globais que foram selecionados para uma área sobre $o$ Nordeste do Brasil. Estes dados estão em pontos de grade $0,144^{\circ}$ de latitude e longitude. Maiores detalhes sobre esses dados podem ser encontrados em Kogan e Sullivan (1993).

As variáveis usadas desse banco de dados NOAANESDIS foram NDVI (Índice de Vegetação de Diferença Normalizada), VCI (índice de condição da vegetação) e TCI (Índice de Condição de Temperatura). O índice de vegetação NDVI é uma razão ou diferença dos valores de reflectância da faixa infravermelho próximo $(0,725$ a 1,10 $\mu \mathrm{m})$ e da faixa visível $(0,4$ a $0,7 \mu \mathrm{m})$ e varia de +1 a -1 .

$$
N D V I=\frac{I R-V I S}{I R+V I S}
$$

O VCI foi derivado do NDVI, que é a proporção da diferença entre a radiação no infravermelho próximo medido pelo AVHRR e a reflectância no canal visível. 


$$
V C I=\frac{(N D V I-N D V I m i n)}{(N D V I m a x-N D V I m i n)} X 100
$$

onde NDVImax, e NDVImin são composições decendiais (10 dias) de NDVI.

O TCI foi derivado das radiações medidas com AVHRR (Advanced Very High Resolution Radiometer) de 10,3 a $11,3 \mu \mathrm{m}$, convertidas em temperatura de brilho (BT). Algoritmos foram desenvolvidos para reduzir o ruído e para ajustar NDVI e BT para a não-homogeneidade da superfície terrestre.

$$
T C I=\frac{(\operatorname{Tmax}-T)}{(\operatorname{Tmax}-\operatorname{Tmin})} X 100
$$

Em toda a série analisada (1982 a 2011) foram retirados os valores extremos e absolutos de cada pixel, isto é, seus valores máximos e mínimos absolutos, respectivamente; T, Tmax, e Tmin são valores para a temperatura de superfície. O VCI e o TCI foram usados para determinar o estresse da vegetação relacionada à água e à temperatura que ocorre durante uma seca ou estiagem (Kogan,1997).

Os dados observados de temperatura do ar a superfície foram obtidos de estações meteorológicas de superfície do Instituto Nacional de Meteorologia (INMET).

A metodologia usada seguiu os seguintes passos: Cálculo sobre o Nordeste do Brasil de médias mensais (janeiro a dezembro) e sazonais (estações do ano: Verão, Outono, Inverno, Primavera) das variáveis do banco de dados da NOAA-NESDIS para o período de 1982-2011; Cálculo de anomalias sazonais para alguns anos de contrastes para os períodos de La Niña, El Niño e Normais das variáveis do banco de dados da NOAA-NESDIS; Cálculo de correlações e plotagens de diagramas de dispersão mensais entre o NDVI e os índices TCI e VCI do banco de dados NOAA-NESDIS; Foi calculada uma média de um retângulo (extrapolando um ponto de grade $0,14^{\circ}$ ) em torno da coordenada geográfica da estação meteorológica que fez as observações das variáveis com os dados do bando de dados NOAA-NESDIS.
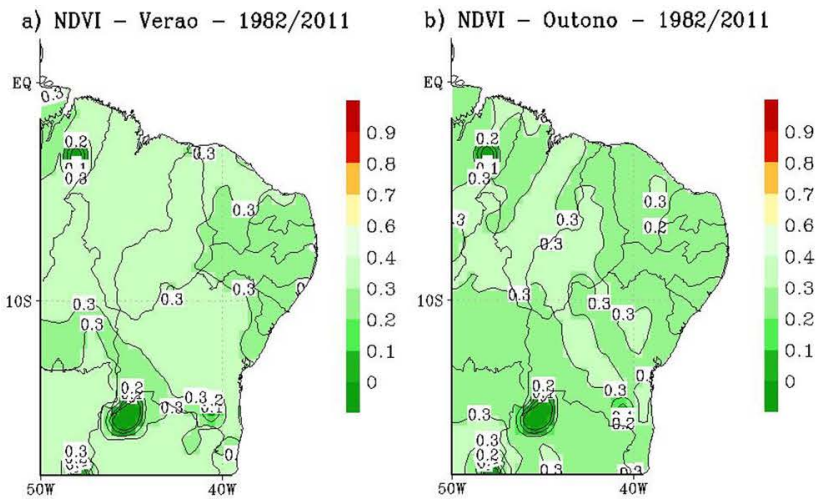

O coeficiente de correlação de Pearson $(r)$ que descreve o grau de colinearidade entre os dados simulados e observados foi usado como comparação, o teste $t$-Student que avalia o nível de significância observada nas correlações foi usado como medida de significância do valor do coeficiente de correlação no nível de 95 usado para aceitar ou rejeitar no teste de hipótese. Os valores de $X$ e $Y$ nas fórmulas representam as variáveis a serem comparadas, (DEVORE, 2006).

Os dados foram compilados em rotinas Fortran disponibilizados em gráficos gerados pelo software GrADS (Grid Analysis and Display System), que é uma ferramenta interativa usada para manipulação e visualização de dados de ciências da terra que usa um ambiente de dados de até 4 dimensões: longitude, latitude, nível vertical e tempo.

\section{Resultados e Discussão}

A variabilidade espacial média de NDVI sazonal sobre o NEB foi representada nas imagens da Fig. 1 utilizando uma série histórica de 1982 a 2011. Nota-se um pequeno decréscimo dos valores NDVIs comparado ao período do outono e inverno, estações em geral de mais chuvas na região. No inverno sobre o NEB o aumento pode ser a resposta média da vegetação as chuvas de outono sobre a região. Nesta escala valores tendendo a zero correspondem a áreas com baixos valores de NDVI.

Na Fig. 2 foi analisada a média espacial da diferença do NDVI sobre o do NEB para as estações de verão, outono, inverno e primavera para anos de contrastes climáticos de El Niño, La Niña e Normais na série histórica de 1982 a 2011. Após análise dos dados da diferença do NDVI sazonal em relação ao NDVI médio nos anos de contrastes climáticos, foi verificado conforme a Fig. 2, que nos anos de El Ninõ houve um predomínio de valores negativos, indicando uma resposta do ambiente na região, o que se pode inferir em características de uma vegetação mais estressada, consistente com uma maior irregularidade da precipitação na região nesses anos de El Ninõ. Ainda verificado na Fig. 2, pode-se perceber que quando o NDVI
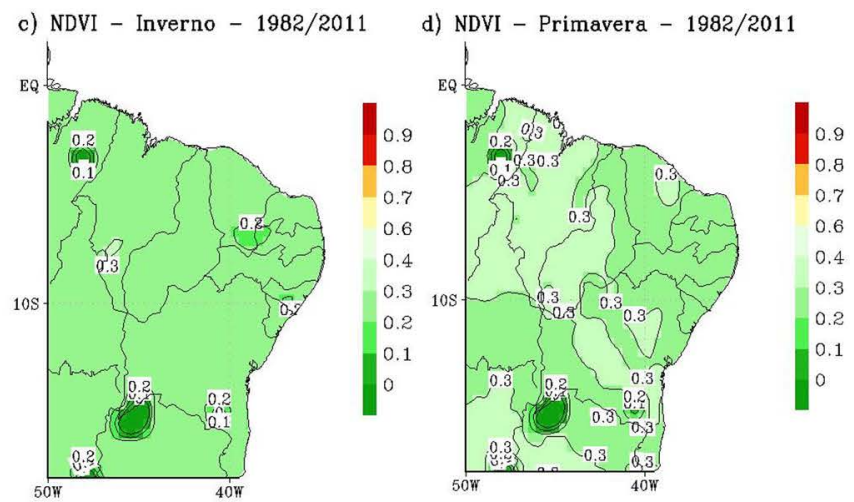

Figura 1 - Variabilidade média espacial sazonal do NDVI: a) Verão, b) Outono, c) Inverno e d) Primavera. 

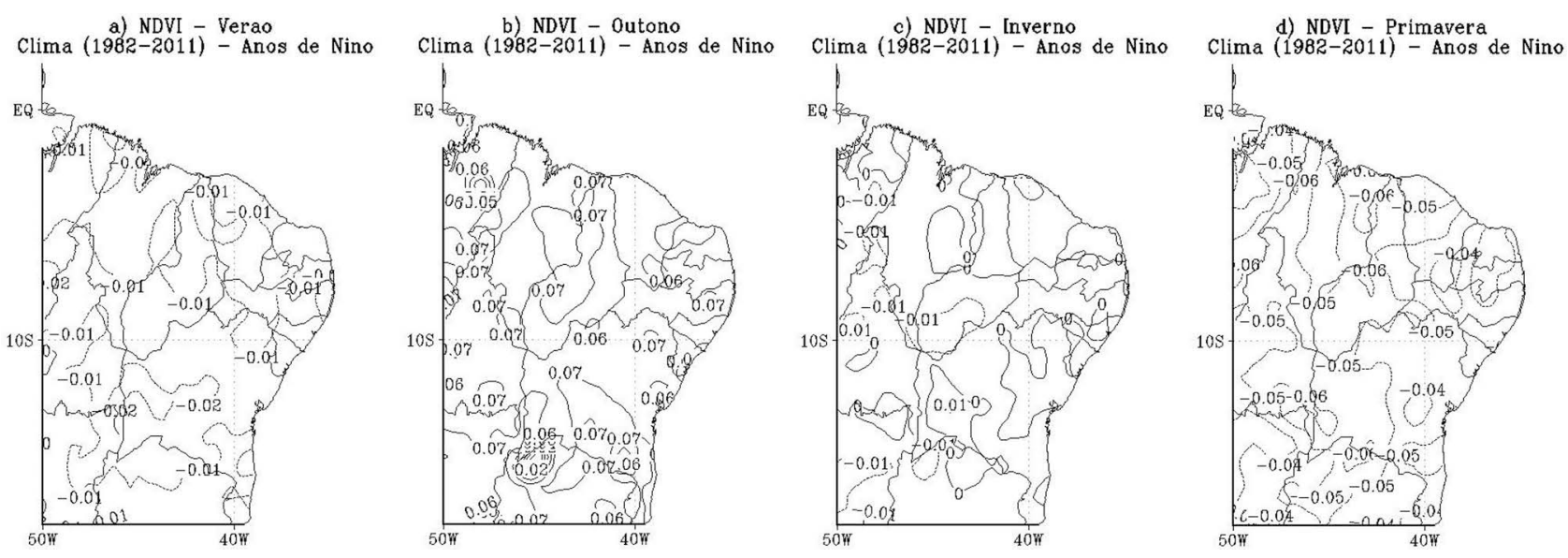

Figura 2 - Variabilidade média espacial da diferença entre o NDVI (1982-2011) e a composição para anos El Niño, a) verão, b) outono, c) inverno e d) primavera.

adquire valores negativos, há forte indicação de vegetação abaixo da condição normal, o que pode ser compreendido em falta de umidade, ou seja, estação de seca na região (BARBOSA, 2004).

Para anos de La Niña, conforme Fig. 3, pode-se observar que houve um predomínio em valores positivos, o que infere em características de uma vegetação menos estressada, consistente com uma menor irregularidade na precipitação da região NEB (Alves, 2004).

Para os anos Normais conforme visto na Fig. 4 as características nas quatro estações é mais similar aos anos de El Niño, pois houve também um predomínio de valores negativos, que infere em características de uma vegetação mais estressada. Pode-se observar pelas Fig. 2, 3 e 4 que as diferenças apresentam um valor muito pequeno, porém em particular nas estações de inverno, primavera e verão em que existe um contraste principalmente entre os anos de El Niño e La Niña (Alves, 2004).

Foi analisada pela Fig. 5 a média espacial da diferença do VCI-TCI (Índice de Condição Vegetativa e índice de Condição de Temperatura) sobre o NEB para as estações de verão, outono, inverno e primavera. Segundo os estudos de Kogan, (1997) e Bhuyian, (2004), a diferença entre: os índices VCI e TCI, pode resultar em boa indicação da variabilidade das condições de vegetação associadas às condições de umidade e de temperatura em uma dada a região. A Fig. 5 mostra uma análise climatológica (1982-2011), espacial sobre o NEB, dessa diferença nas estações de verão, outono, inverno e primavera.

O VCI e TCI são interpretados de formas diferentes. O VCI deve ser interpretado na forma direta, pois valores baixos de VCI resultam em condições de estresse na vegetação. Na avaliação do TCI quanto mais baixos seus valores, estes indicam valores altos e extremos de temperatura da superfície. Dessa forma, esses índices indicam condições de estresse térmico conforme Kogan (1997) Nesta escala valores tendendo a zero correspondem a áreas com baixos valores de NDVI.

Nota-se ainda pela Fig. 5, como mostrado nas análises anteriores, que a variabilidade climática sazonal da
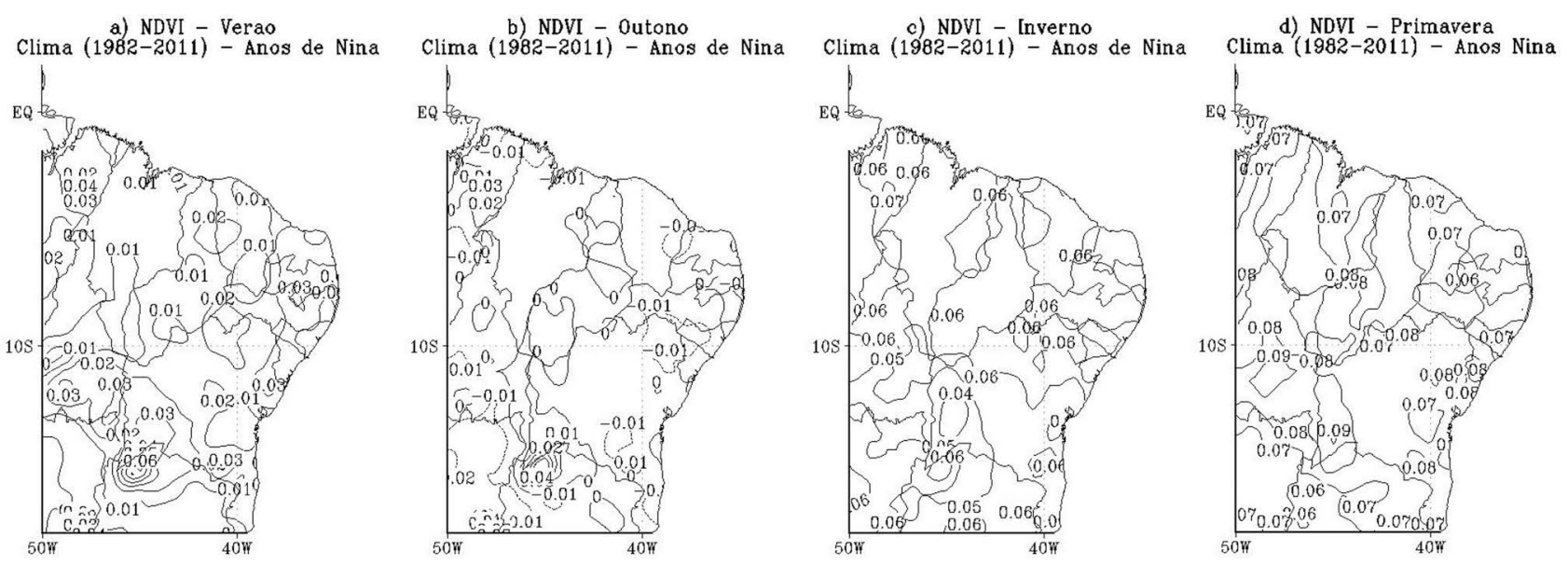

Figura 3 - Variabilidade média espacial da diferença entre o NDVI (1982-2011) e a composição de anos La Niña, a) verão, b) outono, c) inverno e d) primavera. 

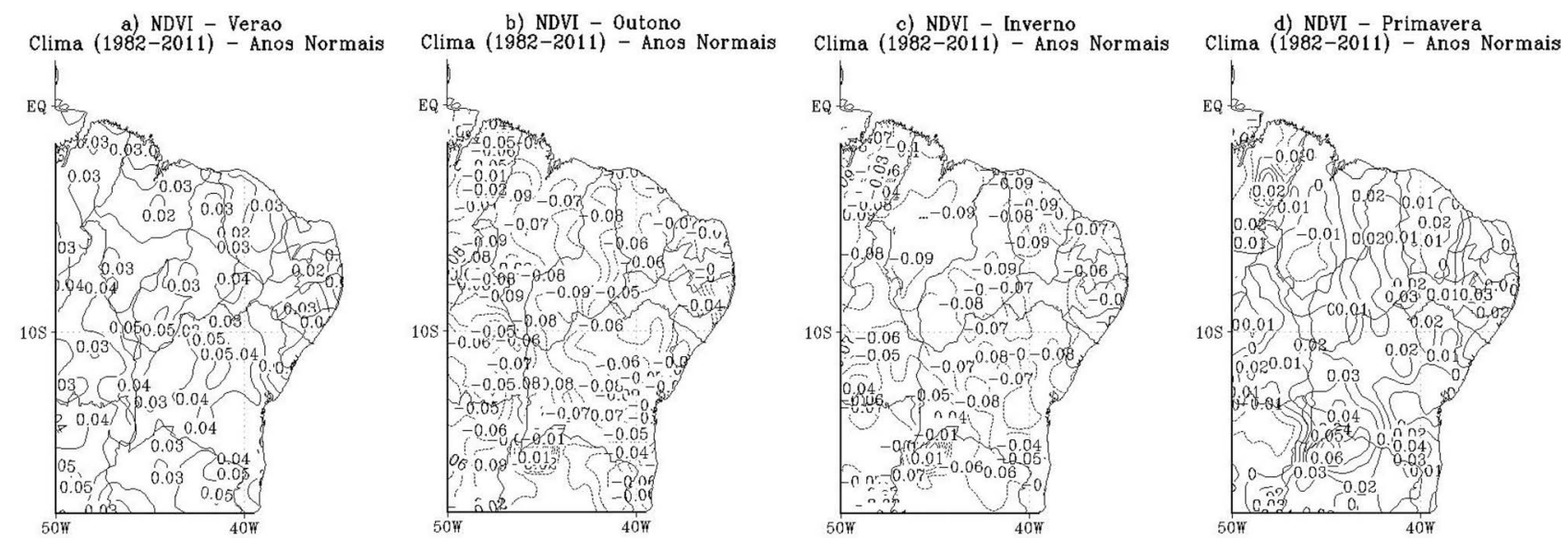

Figura 4 - Variabilidade média espacial da diferença entre o NDV I (1982-2011) e a composição de anos Normais, a) verão, b) outono, c) inverno e d) primavera.
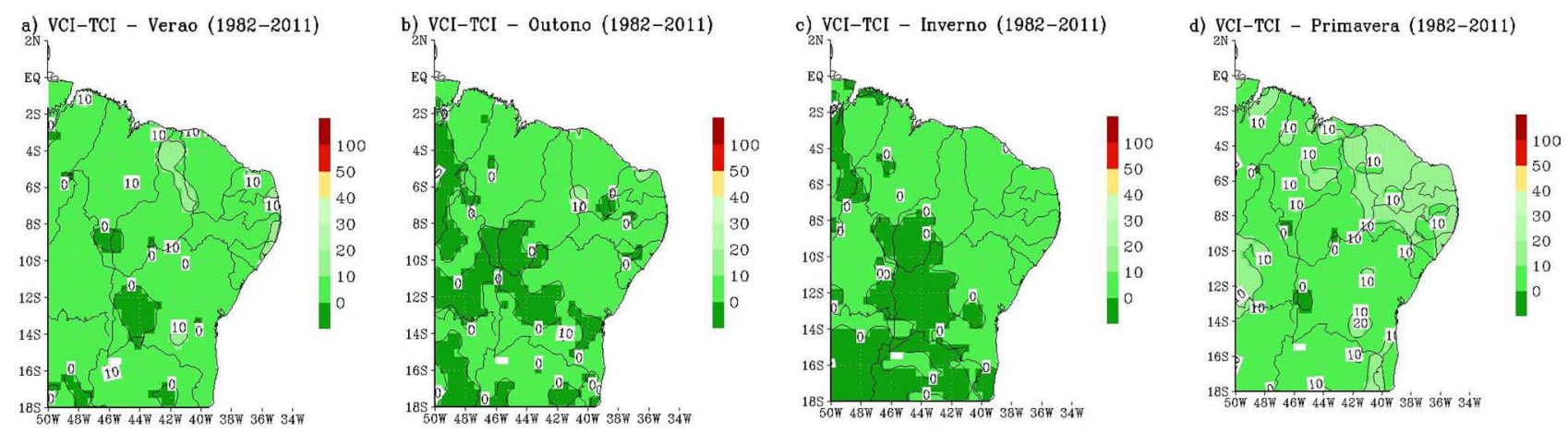

Figura 5 - Variabilidade média espacial da diferença entre o VCI-TCI (1982-2011) sazonalmente: a) verão, b) outono, c) inverno e d) primavera.

diferença entre o VCI e o TCI entre as estações, não é grande sobre o NEB. Os valores dessa diferença oscilam em torno 10 a 0 . Quanto mais próximo de zero mais estressada esta as condições de vegetação. Esses valores da diferença em torno de dez a zero indicam uma condição de vegetação com maior estresse por condição hídrica e altas temperaturas. Em geral, em regiões mais extensas e com áreas mais desérticas como Sudeste Asiático e sobre centro-sul dos Estados Unidos, essa diferença aparece mais relacionada à vegetação estressada nessas áreas desérticas ou semidesérticas e áreas adjacentes mais úmidas (Kogan, 1997).

Essa característica demonstra que climatologicamente embora o NEB, em particular na região semiárida, tenha anos que alternam entre secas e cheias, (o impacto das secas é evidente em todas as regiões do NEB), não aparece uma grande diferença desse estresse climatológico da vegetação sazonalmente no NEB.

Foram analisadas as médias de VCI, TCI e NDVI separadamente nas sub-regiões do nordeste do Brasil nos anos de contrastes climáticos (El Niño, La Niña e Normais). Nos gráficos como pode ser visto nas Figs. 6, 7 e 8 são observadas as variações mensais médias na série histórica de 1982 a 2011 nas sub-regiões do NEB (semiárida do NEB $-2^{\circ} \mathrm{S}-12^{\circ} \mathrm{S}$ e $45^{\circ} \mathrm{W}-37^{\circ} \mathrm{W}$, leste do NEB $-2^{\circ}$ $\mathrm{S}-15^{\circ} \mathrm{S}$ e $37^{\circ} \mathrm{W}-34^{\circ} \mathrm{W}$ e sul do NEB $-15^{\circ} \mathrm{S}-20^{\circ} \mathrm{S}$ e $50^{\circ}$ $\mathrm{W}-34^{\circ} \mathrm{W}$ ). Para os resultados de TCI nota-se que nas três regiões a variabilidade mensal maior, em média, aparece nos anos de El Niño e La Niña conforme Fig. 8. Valores observados de TCI, nos anos de El Niño, mostram entre os meses de maio a novembro, apresentam valores relacionados a uma condição com ambiente de vegetação de mais estresse, com valores menores do que 40 (Kogan, 1996 e 1997).

Para a condição climatológica, essa variabilidade é menor, porém os valores TCI que se associa a uma condição de vegetação e altas temperaturas com menor e maior estresse é menos evidente. Nos anos de La Niña a condição é inversa, refletindo nas três regiões uma condição de maior incidência e regularidade de chuva. Ressalta-se que, na maior parte do ano, os valores de TCI são superiores a 100, condições que infere uma maior umidade e uma condição associada de vegetação mais expressiva e verde predominando em geral de março a outubro. Já nos anos Normais a variabilidade é menor ao longo dos meses do ano. Valores de TCI oscilando entre 40 e 60, o que 

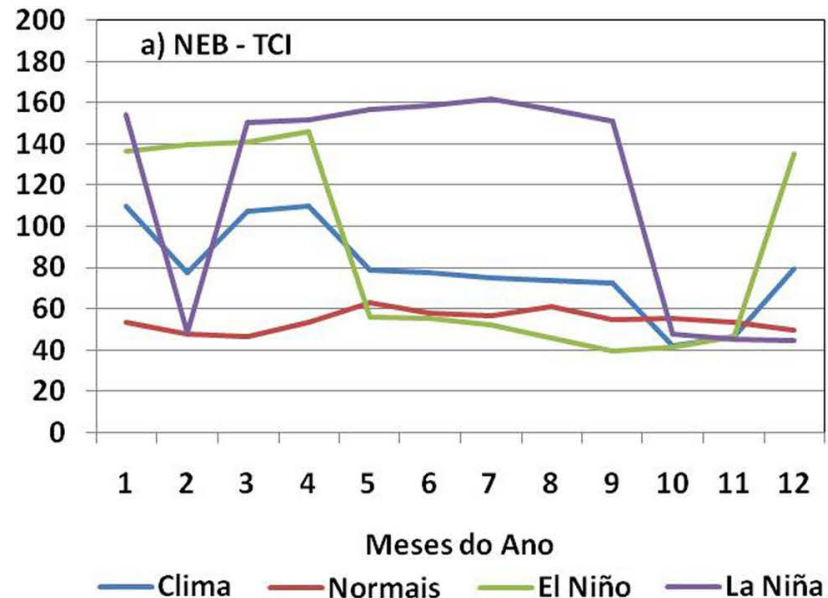

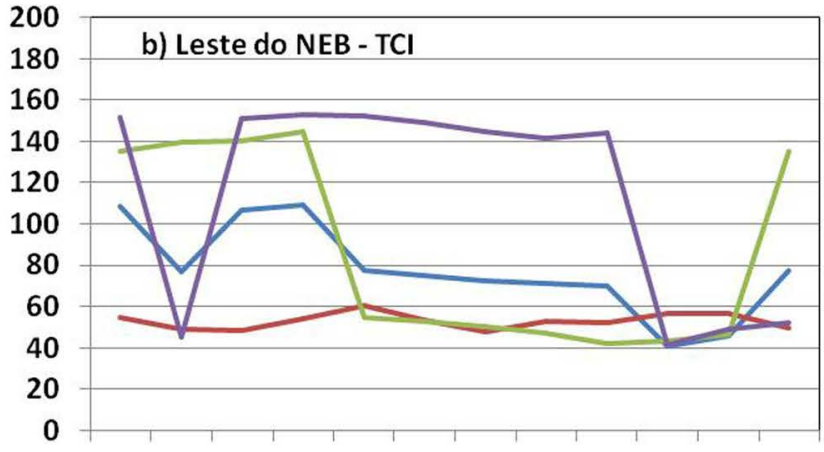

$\begin{array}{llllllllllll}1 & 2 & 3 & 4 & 5 & 6 & 7 & 8 & 9 & 10 & 11 & 12\end{array}$

Meses do Ano

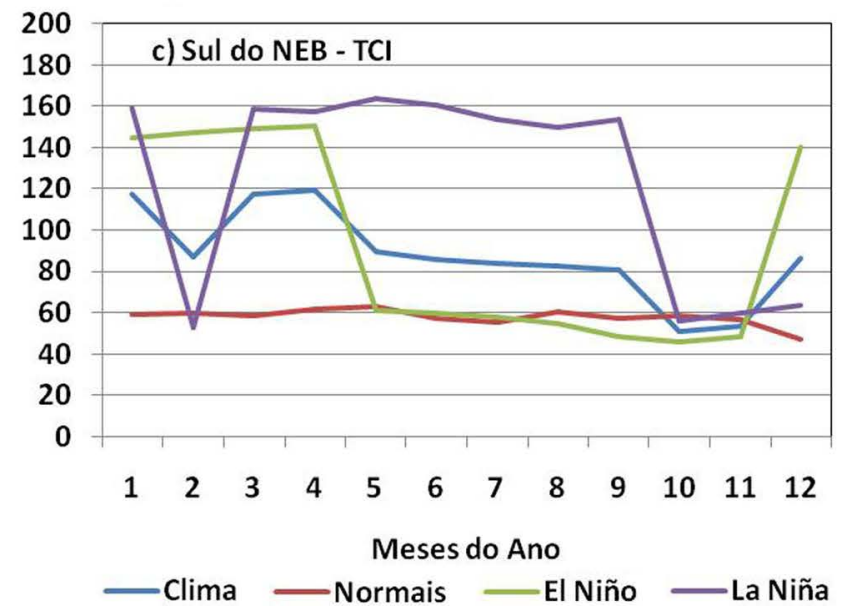

Figura 6 - Variabilidade média mensal de TCI (1982-2011) para anos de El Niño, Normais, e de La Niña: a) Região semiárida do NEB, b) Região Leste do NEB e c) Região sul do NEB.

segundo Kogan (1997) é uma condição intermediária o que infere na condição de vegetação entre menos estresse a uma condição mais robusta.

No gráfico da Fig. 7 pode-se verificar que climatologicamente (1982-2011), a variabilidade para o indice VCI, que reflete nas condições da vegetação, tem influência das condições térmicas do meio ambiente na região, nos anos de El Niño, La Niña e Normais, essa configuração foi aparentemente similar ao longo dos meses na variabilidade do índice TCI. As curvas de variabilidade são quase idênticas apresentando pequenas diferenças para todas as regiões.

Como pode ser visto no gráfico da Fig. 8, foi analisada a variabilidade média do NDVI nos anos de El Niño, La Niña e Normais, e refletem também as variações desses índices TCI e VCI analisados anteriormente para as três regiões em estudo. O NDVI embora não variando significativamente apresenta para as três regiões do NEB, caracaterísticas similares. Nos anos de El Niños e durante a série histórica de 1982 a 2002, houve um aumento de valores do NDVI dos primeiros meses do ano para os meses do final do ano, o que infere a resposta sazonal, em média, nessas áreas da passagem do período mais chuvoso para um período mais seco. Nos anos de La Niña essa característica não é evidente há uma maior variabilidade média mensal. Nos anos classificados como Normais em todas as regiões ocorreu um aumento do NDVI entre setembro e janeiro e um pequeno decréscimo entre fevereiro a agosto.

A Fig. 9 mostra a média da anomalia normalizada pela média mensal nos meses de dezembro e janeiro (1982-2011) do NDVI médio e os índices TCI e VCI nas sub-regiões semiárida do NEB, leste do NEB e sul do NEB. Essa análise foi feita pela plotagem de um diagrama de dispersão e a inferência da curva de tendência e o coefiente de correlação ao quadrado. A medida de ajustamento do modelo estatístico linear utilizado deverá indicar uma percentagem que varia de 0 a 1 , sendo assim um percentual de $\mathrm{R}^{2}$ alto no gráfico indicará que o modelo conseguiu explicar os valores observados. Quanto menor o percentual menos explicativo é o modelo, e menos ele se ajusta à amostra como pode ser observado nos resultados acima. 


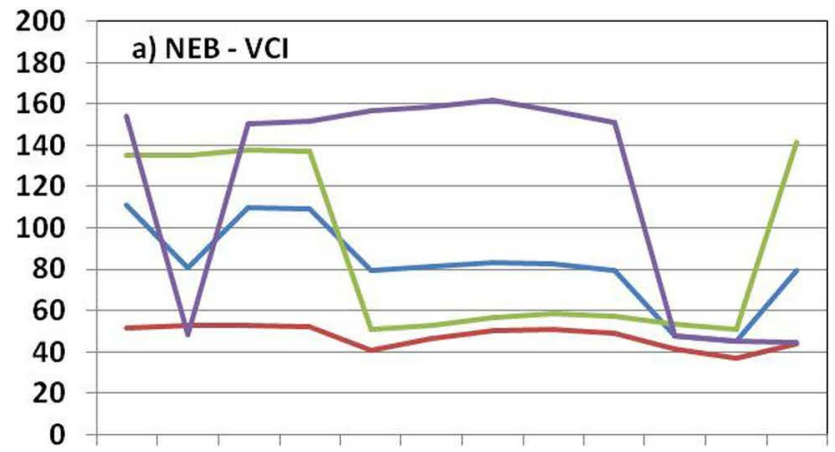

$\begin{array}{llllllllllll}1 & 2 & 3 & 4 & 5 & 6 & 7 & 8 & 9 & 10 & 11 & 12\end{array}$

Meses do Ano
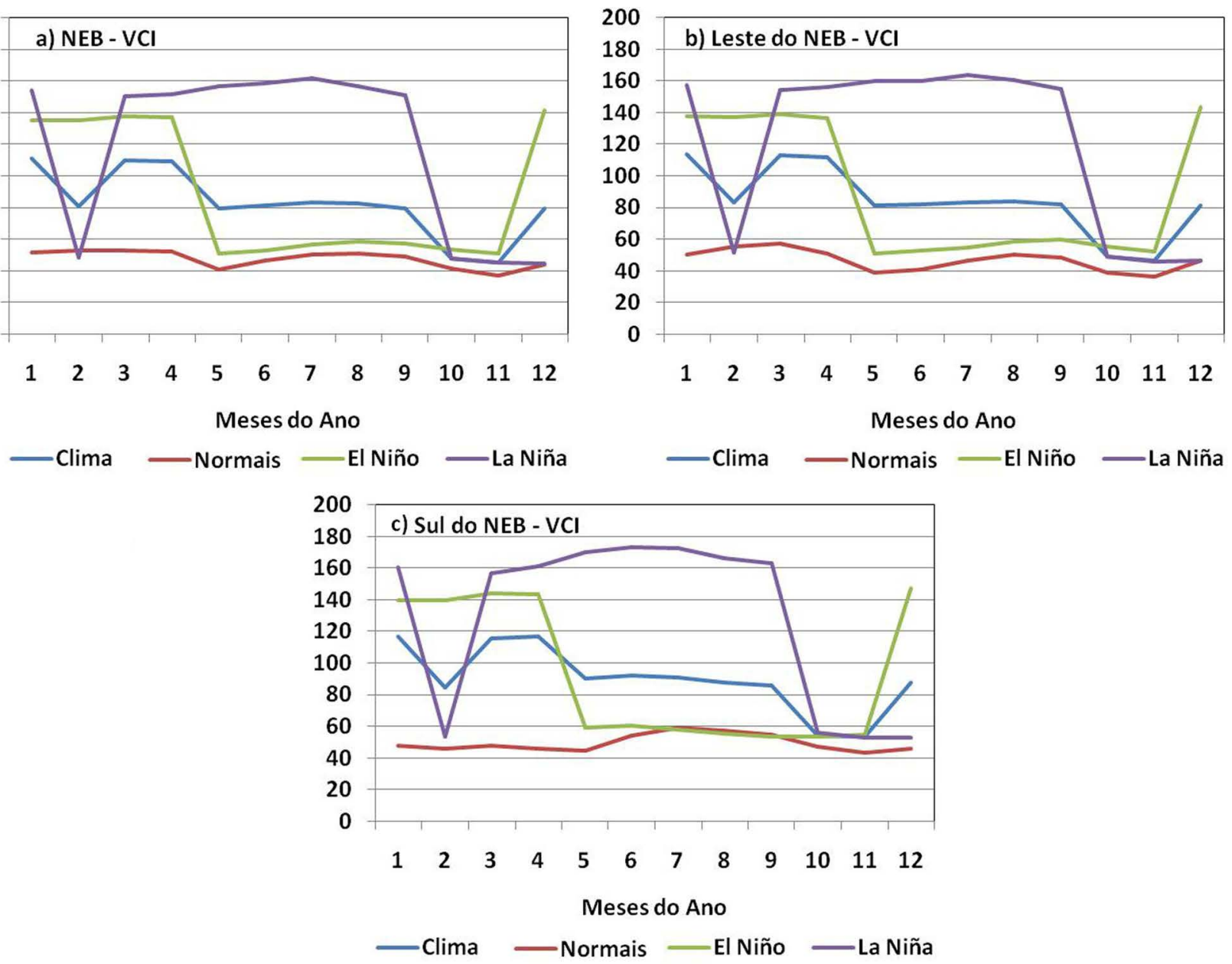

Figura 7 - Variabilidade média do VCI (1982-2011) e pra anos de El Niño, Normais, e de La Niña: a) Região semiárida do NEB, b) Região Leste do NEB e c) Região sul do NEB.

Nota-se na Fig. 9 que a relação entre NDVI e os índices TCI é predominantemente linear inversa. Isto é, quando os valores de TCI aumentam o NDVI tende a diminuir. Fisicamente os índices de TCI quando tendem a valores baixos e VCI quando tendem a mostrar valores muitos altos, implicam em condições de vegetação menos estressada a ótima, nesse caso o NDVI tem valores maiores, consistente com essa condição de vegetação sem estresse. Essas características se repetem para as demais sub-regiões do NEB. A explicação da variância linear, isto é, o que cada índice TCI e VCI explicam da variabilidade do NDVI para a região semiárida do NEB foi de $0,56 \%$ para o TCI e $0,61 \%$ para VCI, para o leste do NEB essa explicação da variância foi de $0,52 \%$ para TCI e $0,50 \%$ para o VCI, enquanto para o sul do NEB essas variâncias foram de $0,41 \%$ e $0,33 \%$, a menor explicação de todas as regiões.

\section{Conclusões}

No período de 1982 a 2011 foi analisada a média espacial da diferença do NDVI sazonal (verão, outono, inverno e primavera) sobre o NEB para anos de contrastes climáticos de EL NIÑO, LA NIÑA e NORMAIS, os resultados mostraram que nessa região predominam características de uma vegetação estressada, devido à irregularidade da precipitação na região. Essa característica (condição de vegetação com maior estresse por condição hídrica e altas temperaturas) também foi confirmada através da média espacial da diferença do VCI-TCI sobre o do NEB para as estações de verão, outono, inverno e primavera na série histórica (Fig. 5).

As tendências crescentes e positivas de NDVI encontradas podem ser causadas devido ao aumento correspondente desde a ocorrência de chuva (quadra chuvosa) e aumento de umidade no solo, que se mantem nas estações e pós-estações chuvosa de crescimento da vegetação como resposta a precipitação.

Foram analisadas as médias do VCI, TCI e NDVI para as sub-regiões semiáridas do NEB, leste do NEB e sul do NEB, nas Figs. 6, 7 e 8 para os anos de El Niño e La Niña e normais. Verificou-se que a variabilidade média de TCI nos anos de El Niño, entre os meses de maio a 

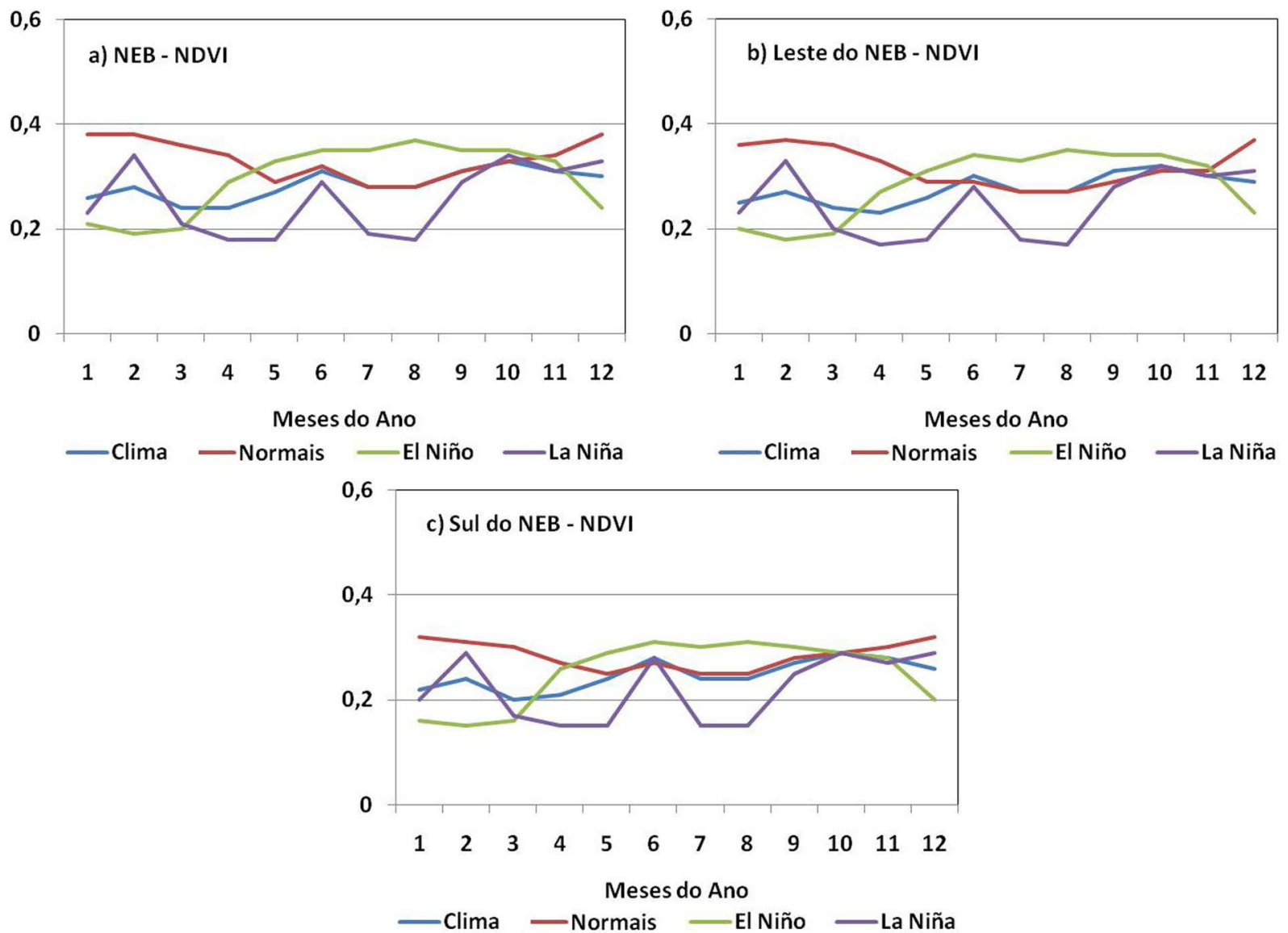

Figura 8 - Variabilidade média do NDVI (1982-2011) para os anos de El Niño, Normais, e de La Niña: a) Região semiárida do NEB, b) Região Leste do NEB e c) Região sul do NEB.

novembro, foram relacionadas a uma condição com ambiente de vegetação de mais estresse (menor que 40). Para o TCI nos anos de La Niña a condição é inversa, refletindo nas três regiões uma condição de maior incidência e regularidade de chuva (acima de 60), Nos anos Normais a variabilidade foi menor ao longo dos meses do ano (entre 40 e 60). O indice VCI para os anos de El Niño, La Niña e Normais, obtiveram configuração similar ao longo dos meses, com a variabilidade do índice TCI apresentando apenas pequenas diferenças.

Nos anos de El Niño houve um aumento dos valores do NDVI nos primeiros meses do ano e para os meses do final do ano, o que infere em uma resposta sazonal, nessas áreas da passagem do período mais chuvoso para um período mais seco. Nos anos de La Niña essa característica não foi evidente devido a maior variabilidade média mensal do NVDI, por causa da precipitação. Nos anos classificados como Normais em todas as regiões do NEB, ocorreu um aumento do NDVI entre setembro e janeiro e um pequeno decréscimo entre fevereiro a agosto.

Quando analisada a relação linear entre NDVI e VCI, TCI nas sub-regiões semiáridas do NEB, leste do NEB e sul do NEB conforme Fig. 9, fica evidente que a variância linear, do índice TCI e VCI relacionado à variabilidade do NDVI nessas áreas foi maior para o semiárido do NEB $(0,56 \%$ para o TCI e $0,61 \%$ para $\mathrm{VCI})$ o leste do NEB ficou em $0,52 \%$ para TCI e $0,50 \%$ para o VCI e o sul do NEB o resultado foi de $0,41 \%$ TCI e $0,33 \%$ VCI indicando a menor explicação de todas as regiões. O NDVI é controlado pela umidade, além de outros fatores que podem causar alterações aparentes na leitura da vegetação, podendo haver ruidos que possam infeir em incertezas associada aos registros NDVI e sua suscetibilidade e sensibilidade aos efeitos atmosféricos. Santos e Negri (1996) mostraram que para regiões semiáridas como o NEB, áreas com precipitação de até $50 \mathrm{~mm} /$ mês possuem uma relação linear mais forte e mais evidente do NDVI com a precipitação. Quando esse total de precipitação é mais excessivo essa relação não é tão percepitivel. Sendo assim podemos inferir que para as sub-regiões do NEB o valores de VCI e o TCI e NDVI demonstraram valores expressivos nesse estudo, porém alguns resultados quando avaliados isoladamente para algumas áreas da região $\mathrm{NEB}$, não foram capazes de explicar à dinâmica e condições nessas áreas, sendo necessária uma análise conjunta com outros índices que possam minimizar o ruído. 

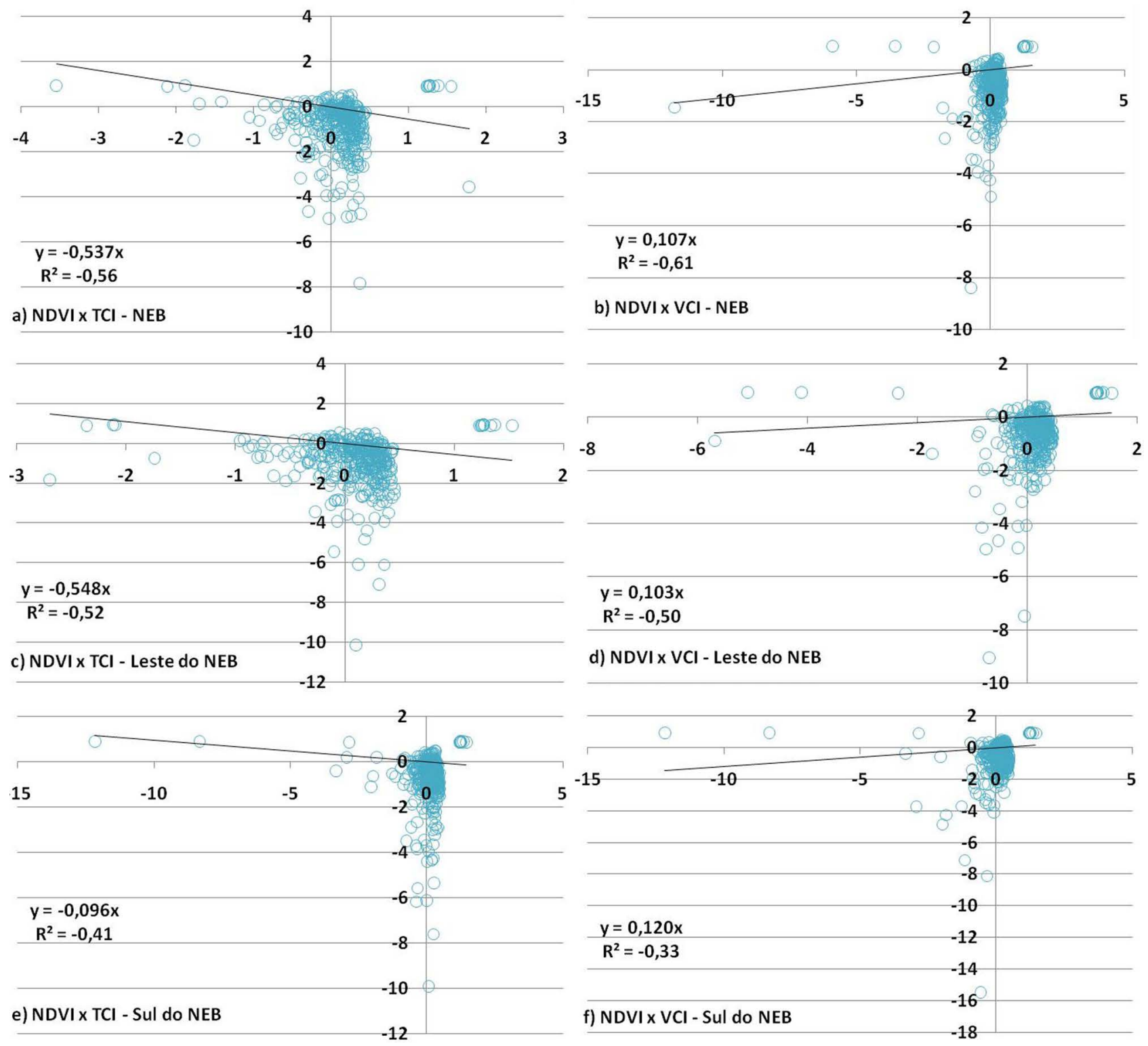

Figura 9 - Relação linear entre o NDVI e os índices TCI e VCI (1982-2011): a) Reg. semiárida do NEB NDVI x TCI, b) Reg. semiárida do NEB NDVI x VCI, c) Reg. leste do NEB NDVI x TCI, d) Reg. leste do NEB NDVI x TCI, e) Reg. sul do NEB NDVI x TCI e f) Reg. Leste do NEB NDVI x VCI.

\section{Agradecimentos}

Agradecimento a Universidade Estadual do Ceará pela criação do curso de Mestrado Profissional em Climatologia e países da CPLP e África, a coordenação do curso e aos professores que estiveram presente dando apoio durante todo o percurso. Este artigo é parte da Dissertação de Mestrado da primeira autora apresentada no Mestrado Profissional em Climatologia de Países da Língua Portuguesa da Universidade Estadual do Ceará (UECE).

\section{Referências}

ALVES, J.M.B.; COSTA, A.A.; FERREIRA, F.F. Um estudo dos impactos das Temperaturas da Superfície do Mar (TSM) em macrorregiões, pluviometricamente homogêneas no Estado do Ceará. Revista Brasileira de Meteorologia, v. 12, n. 1, p. 193-204, 2004.

ARAUJO, M.L.; RODRIGUES J.E.C.; LUZ L.M. Cobertura vegetal em áreas urbanas: a perda da cobertura vegetal e uso do solo no bairro do Tenoné - Belém/PA. Belém: IFPA, 2011. Disponível em: https://www.escavador.com/ sobre/3684817/marlisson-lopes-de-araujo, acesso 12 mai. 2016.

BARBOSA, H.A. Vegetation dynamics over the Northeast region of Brazil and their connections with climate variability during the last two decades of the twentieth century. Tucson, Arizona, 2004, Disponível em: https:// www.sciencedirect.com /science/article/pii/ S0140196306000796, acesso em: 23 abr. 2016. 
BHUIYAN, C. Various drought indices for monitoring drought condition in Aravalli terrain of India, In: Proceedings of the XXth ISPRS Conference, Int. Soc. Photogrammetry and Remote Sensing, Istanbul, 2004. Disponivel em: http:// www.isprs.org/proceedings/XXXV/congress/comm7/ papers/243.pdf, acesso em: 13 abr. 2017.

CHIANG, J.C.H.; KUSHNIR, Y.; ZEBIAK, S.E. Interdecenal changes in castern Pacific ITCZ variability and its influence on the Atlantic ITCZ. Geophysical Research Letters, v. 27, p. 3687-3690, 2000.

DEVORE, J.L, Probabilidade e estatística: para engenharia e ciências. Tradução: Joaquim Pinheiro Nunes da Silva, 6 edição. São Paulo: Edit. Pioneira Thomson Learning, p. 682, 2006.

FERREIRA, N.S. Zona de Convergência Intertropical. In: Climanálise Boletim de Monitoramento e Analise Climática. Edição Especial de $\mathbf{1 0}$ anos. Cachoeira Paulista: MCT/INPE, p. 235, 1996.

KOGAN, J., AND SULLIVAN, J., Development of global drought-watch system using NOAA/AVHRR data NOAA/ NESDIS/ORA/Satellite Research Laboratory. Advances in Space Research, v. 13, n. 5, p. 219-222, 1993.

KOGAN, J.T.; SULLIVAN, P.B.C., Testing post-launch calibration for the AVHRR sensor on world desert targets during 1985-1993. Adv. Space Res., v. 17, n. 1, p. 47-50, 1996.

KOGAN, J. Global drought watch from space. Bulletin of American Meteorology Society, v. 78, n. 4, p. 621-636, 1997.
RAO, V.B.; LIMA, M.C.D.; FRANCHITO, S.H. Seasonal and Interannual variations of rainfall over eastern Northeast Brazil. Journal of Climate, v 6, p. 1754-1763, 1993.

SANTOS, P.; NEGRI, A. A Comparison of the Normalized Difference Vegetation Index and Rainfall for the Amazon and Northeastern Brazil. Journal Applied of Meteorology, v. 26, p. 958-965, 1996.

UVO, C.R.B.; BERNDTSSON, R., Regionalization and spatial properties of ceará state rainfall in Northeast Brazil. Journal of Geophysical Research, v. 101, n. D2, p 4221-4233, 1996.

\section{Endereços de Internet}

FUNDAÇÃO CEARENSE DE METEOROLOGIA E RECURSOS HÍDRICOS, FUNCEME, Disponível em: http://www. funceme.br/, acesso em: 9 de maio de 2016.

INSTITUTO NACIONAL DE METEOROLOGIA, INMET. Disponível em: http://www.inmet.gov.br/portal/index.php? $\mathrm{r}=$ home/page\&page $=$ omm, acesso em: 9 mai. 2017.

INSTITUTO NACIONAL DE PESQUISAS ESPACIAIS, INPE. Campo Grande, 1982, Disponível em: http://mtc-m16b.sid. inpe.br/col/sid.inpe.br/jeferson/2005/06.21.17.31/doc/publi cacao.pdf, acesso em 24 Abr. 2017.

License information: This is an open-access article distributed under the terms of the Creative Commons Attribution License (type CC-BY), which permits unrestricted use, distribution and reproduction in any medium, provided the original article is properly cited. 